\title{
Complexity analysis of respiratory dynamics
}

Mohammad Reza Raoufy ${ }^{1,2^{*}}$, Tara Ghafari ${ }^{3}$, Ali R. Mani ${ }^{4}$

1. Department of Physiology, Faculty of Medical Sciences, Tarbiat Modares University, Tehran, Iran

2. Chronic Respiratory Diseases Research Center (CRDRC), National Research Institute of Tuberculosis and Lung Diseases (NRITLD), Shahid Beheshti University of Medical Sciences, Tehran, Iran

3. Department of Physiology, Medical School, Shahid Beheshti University of Medical Sciences, Tehran, Iran

4. Division of Medicine, Royal Free Campus, University College London, London, UK

* Corresponding author: Mohammad Reza Raoufy (MD, PhD) Department of Physiology, Faculty of Medical Sciences, Tarbiat Modares University, Nasr Bridge, Jalal Al Ahmad Highway, Tehran, Iran. PO Box: 14115-331 Phone: +98-21-82884586 Fax: +98-21-82884528 Email: $\underline{\text { raoufy@modares.ac.ir }}$

\section{Letter to Editor}

We read with interest the article by Thamrin and colleagues (1), which discussed systems biology approaches to study respiratory system, as a complex system, within the context of diagnosis and monitoring of diseases. A section of the article described previous studies that characterized the complex behavior of the lung in respiratory diseases using nonlinear analysis of the fluctuations of physiologic variables over time. Chronic lung diseases have variable clinical symptoms and represent complex behavior which may be associated with a shift in dynamics of respiratory system toward either too regular or too irregular (2). Analyzing such nonlinear fluctuations may not only improve traditional assessments in diagnosing the onset of illness, its severity and prognosis (1), but also could provide new insights into the different pathophysiological characteristics of respiratory diseases (2). In addition to studies described by Thamrin and colleagues (1), our recent report (2) showed that both respiratory rhythm and volume fluctuate continuously under a delicate equilibrium to maintain adaptability to external or internal stimuli. These fluctuations showed decreased long-range correlation, increased regularity and reduced sensitivity to initial conditions in patients with asthma, particularly in uncontrolled state. More importantly, receiver operating characteristic (ROC) curve analysis showed that respiratory variability analysis can be useful not only to aid in asthma diagnosis and in differentiating uncontrolled from controlled asthma but also for discriminating between nonatopic and atopic asthma (2). We found decreased long range correlation, irregularity and chaotic nature of respiratory dynamic in non-atopic asthma as compared with atopic asthma, possibly reflecting different pathophysiological mechanisms and maybe justifying a higher degree of disease severity in patients with 
non-atopic asthma (3). Therefore, complexity analysis of respiratory dynamics may represent a novel physiologic marker to facilitate diagnosis and clinical assessment of respiratory disorders.

In addition, a system biology approach to respiratory system may lead to development of novel indices for evaluation of the respiratory control systems. For instance the "controllability" of physiological systems can be assessed using nonlinear analysis of cardiorespiratory signals $(4,5)$. In the control theory, a controllable system is described as a system that can be driven from its initial inputs to the desired state within a limited time (6). Although well-developed tools are available to calculate controllability in engineered systems, they are not extensively used in physiological systems. Controllability of physiological systems can be estimated indirectly by measurement of memory in physiological timeseries (4). Memory in a time-series (e.g. respiratory rhythm) indicates how many steps we need to go forward to reach a point where the present state does not depend on its past $(7,8)$. Thus controllability is affected by the concept of memory in a time-series; intuitively the control of a system with longer memory is also harder. In this regard, we recently quantified memory in respiratory rhythm of patients with asthma and showed that it is significantly prolonged in patients with uncontrolled asthma in comparison with patients with controlled asthma (8). This suggested that it is more likely that a sudden stochastic event (e.g. tachypnea) is followed by further tachypnic events in patients with uncontrolled asthma. Such approach may explain important dynamical events in pathophysiology of respiratory disease such as air trapping during asthma attacks (8). Thus, low memory and high controllability is needed to respond rapidly to the environmental challenges without keeping the memory of the past stochastic events (8). Using systems biology for developing novel indices for assessment of control and regulation in respiratory system may be beneficial in clinical diagnosis; it can also pave the way in understanding the mechanism of complex respiratory disorders such as uncontrolled asthma.

References

1. Thamrin C, Frey U, Kaminsky DA, Reddel HK, Seely AJ, Suki B, Sterk PJ. Systems Biology and Clinical Practice in Respiratory Medicine. The Twain Shall Meet. Am J Respir Crit Care Med. 2016 Nov 1;194(9):1053-1061.

2. Raoufy MR, Ghafari T, Darooei R, Nazari M, Mahdaviani SA, Eslaminejad AR, Almasnia M, Gharibzadeh S, Mani AR, Hajizadeh S. Classification of Asthma Based on Nonlinear Analysis of Breathing Pattern. PLoS One. 2016 Jan 29;11(1):e0147976.

3. Rackemann FM. A working classification of asthma. Am J Med. 1947 Nov;3(5):601-6.

4. Mazloom R, Shirazi AH, Hajizadeh S, Dehpour AR, Mani AR. The effect of endotoxin on the controllability of cardiac rhythm in rats. Physiol Meas. 2014; 35(3): 339-49.

5. Taghipour M, Eftekhari G, Haddadian Z, Mazloom R, Mani M, Mani AR. Increased sample asymmetry and memory of cardiac time-series following endotoxin administration in cirrhotic rats. Physiol Meas. 2016 Nov;37(11):N96-N104. 
6. Liu YY, Slotine JJ, Barabási AL. Controllability of complex networks. Nature. 2011 May 12;473(7346):167-73.

7. Ebadi $H$, Shirazi AH, Mani AR, Jafari GR. Inverse statistical approach in heartbeat time series. J Stat Mech. 2011 (08), P08014.

8. Shirazi AH, Raoufy MR, Ebadi H, De Rui M, Schiff S, Mazloom R, Hajizadeh S, Gharibzadeh S, Dehpour AR, Amodio $P$, Jafari GR, Montagnese $S$, Mani AR. Quantifying memory in complex physiological timeseries. PLoS One. 2013 Sep 5;8(9):e72854. 